\title{
Habilidades linguísticas: a relação entre a consciência fonológica e a escrita
}

\section{Language skills: the relationship between phonological awareness and writing}

Habilidades lingüísticas: relación entre conciencia fonológica y escritura

\section{Acácia Aparecida Angeli dos Santos*}

Universidade São Francisco - USF, Campinas, São Paulo, Brasil

\section{Adriana Satico Ferraz**}

Universidade São Francisco - USF, Campinas, São Paulo, Brasil

\section{Thatiana Helena de Lima***}

Universidade Federal da Bahia - UFBA, Salvador, Bahia, Brasil

\section{Neide de Brito Cunha****}

Universidade do Vale do Sapucaí - UNIVAS, Pouso Alegre, Minas Gerais, Brasil

\section{Adriana Cristina Boulhoça Suehiro*****}

Universidade Federal do Recôncavo da Bahia - UFRB, Santo Antônio de Jesus, Bahia, Brasil

\section{Katya Luciane de Oliveira******}

Universidade Estadual de Londrina - UEL, Londrina, Paraná, Brasil

\section{Alexandra Ayach Anache******* \\ Universidade Federal de Mato Grosso do Sul - UFMS, Campo Grande, Mato Grosso do Sul, Brasil}

I olete Ribeiro da Silva********

Universidade Federal do Amazonas - UFAM, Manaus, Amazonas, Brasil

\begin{abstract}
RESUMO
O presente estudo objetivou, principalmente, investigar a associação entre a consciência fonológica e a escrita em escolares do ensino fundamental e, adicionalmente, comparar os níveis de consciência fonológica entre os anos escolares, o sexo e regiões de proveniência dos alunos. Participaram 317 crianças, com idades entre sete e 12 anos, matriculadas no $3^{\circ}, 4^{\circ}$ e $5^{\circ}$ anos de escolas em diferentes regiões do Brasil, que responderam ao Roteiro de Avaliação da Consciência Fonológica (RACF) e à Escala de Avaliação da
\end{abstract}


Escrita (EAVE). Os dados mostraram a existência de correlação positiva e significativa entre ambas, assim como revelaram a existência de diferenças, tanto entre os anos escolares como entre as regiões. Os resultados são congruentes com os de outros estudos na área e apontam para a importância de que novas investigações sobre o tema sejam realizadas, visando à consolidação do conhecimento sobre o papel das habilidades linguísticas.

Palavras-chave: aprendizagem, avaliação psicológica, habilidades metalinguísticas.

\begin{abstract}
This study objective investigate the association between phonological awareness and writing in primary students and additionally compare the phonological awareness levels among school years and regions of origin of the participants. Participated 317 children, aged between 7 and 12 years, enrolled in the 3rd, 4th and 5th years of schools from different regions of Brazil, who responded to Roadmap of Phonological Consciousness Evaluation (RACF) and Writing Assessment Scale (EAVE). The data showed the existence of positive and significant correlation between both of, and revealed the existence of differences among school years and between regions. The results are congruent with those of other studies in the area and point out the importance of further research into the subject, to consolidate the knowledge about the role of language skills.
\end{abstract}

Keywords: learning, psychological assessment, metalinguistic skills.

\title{
RESUMEN
}

El principal objetivo de este estudio fue investigar la relación entre la conciencia fonológica y la escritura de alumnos de primaria y, además, comparar los niveles de conciencia fonológica entre los años escolares y regiones de origen de los participantes. Participaron 317 niños, de entre 7 y 12 años, matriculados en el tercero, cuarto y quinto años de escuelas en diferentes regiones de Brasil, que respondieron al Guion de Evaluación de la Conciencia Fonológica (RACF) y la Escala de Evaluación de la Escrita (EAVE). Los datos mostraron la existencia de una correlación positiva y significativa entre ambos y revelaron la existencia de diferencias entre los años escolares y entre las regiones. Los resultados son congruentes con los de otros estudios en el área y señalan la importancia de que nuevas investigaciones sobre el tema se realicen para consolidar el conocimiento sobre el papel de las habilidades lingüísticas.

Palabras clave: aprendizaje, evaluación psicológica, habilidades metalingüísticas.

\section{I ntrodução}

A aquisição da escrita ocorre, frequentemente, durante o período da alfabetização infantil e está atrelada a aspectos maturacionais, socioculturais e a diferenças na didática adotada pelos professores, assim como à estrutura física e pedagógica das escolas, os quais interferem na aprendizagem (Araujo \& Minervino, 2008; Cárnio, Pereira, Alves \& Andrade, 2011; Rego \& Bryant, 1993). Alguns fatores destacam-se na literatura como componentes relacionados à 
aquisição da escrita, incluindo a psicomotricidade, a cognição, as dificuldades relacionadas à apreensão das regras ortográficas e ao domínio da linguagem oral (Araujo \& Minervino, 2008; Mezzomo, Mota, \& Dias, 2010). Embora não se descarte a relevância de cada um desses elementos, este estudo dá maior ênfase aos aspectos relativos às habilidades cognitivas, em especial à consciência fonológica e sua relação com a aprendizagem da escrita. Dessa forma, o seu objetivo principal é verificar como essas habilidades se mostram associadas.

As habilidades cognitivas permitem que o aluno retenha e organize os conceitos aprendidos, por meio da atenção, percepção e memória, para evocá-los posteriormente (Souza \& Sisto, 2001). Nesse sentido, o processamento fonológico se destaca como um importante componente cognitivo, referente às operações mentais de processamento de informação que são fundamentadas na estrutura fonológica da linguagem oral. Autores como Lopes, Lopes, e Gallera (2005) argumentam que o processamento fonológico é responsável por armazenar e manipular toda informação verbal. De forma elucidativa pode-se dizer que a informação é recepcionada e mantida em um armazenador primário (controle articulatório com base na fala). Essa informação passa a ser conservada na memória em razão da reverberação ou mecanismo de recitação. $O$ processamento fonológico é composto por três componentes, a saber, a velocidade de nomeação, a memória de trabalho fonológica e a consciência fonológica (Capovilla, Gütschow, \& Capovilla, 2004).

Como parte integrante do processamento fonológico, a consciência fonológica se refere à habilidade de refletir de forma consciente sobre os sons das palavras, tornando possível a manipulação dos sons da fala (fonemas). Os fonemas representam as menores unidades da fala que atribuem significado a uma palavra (Capovilla et al., 2004; Costa, 2013; Fletcher, Lyons, Fuchs \& Barns, 2009; Valente \& Martins, 2012).

Por sua vez, Sisto e Fernandes (2004) salientam a importância da consciência fonológica para o processo de aprendizagem da escrita, no que se refere ao desenvolvimento de habilidades que possibilitam a conversão dos elementos sonoros das palavras em unidades auditivas, ou seja, transformar as unidades sonoras em representações gráficas análogas por meio de signos. Esse processo revela a importância do domínio da linguagem oral para a aprendizagem da escrita, tendo em vista que ele pode favorecer a sua aquisição (Mezzomo et al., 2010; Pestun, Omote, Barreto \& Matsuo, 2010; Suehiro \& Hohlenwerger, 2014).

Para Capovilla et al. (2004), déficits relacionados à consciência fonológica podem gerar dificuldades no processo de aprendizagem da leitura e escrita. Se não detectados a tempo e devidamente trabalhados por meio de estimulação da consciência fonológica 
podem implicar em danos maiores, que remetem à automatização dos erros expressos na escrita e leitura incorreta das palavras, os quais na maioria das vezes implicam no baixo rendimento escolar.

No que se refere à escrita, autores apontam para a perspectiva fonológica como um modelo associado a sua aprendizagem, com ênfase para o conhecimento da correspondência do som das letras (Cardoso-Martins, 2013; Cardoso-Martins \& Corrêa, 2008; Pollo, Treiman, \& Kessler, 2015; Valente \& Martins, 2012). Tal modelo é composto por quatro fases conforme o modelo de Linnea Ehri, sendo dividido em fase pré-alfabética, semialfabética, alfabética completa e alfabética consolidada (Cardoso-Martins \& Corrêa, 2008).

Na primeira fase a criança não detém o conhecimento sobre o nome das letras e seus respectivos sons. Portanto, a tentativa de escrita é expressa de forma arbitrária, ou seja, sem relação entre os sons das palavras. Na fase semialfabética, passa a compreender que há uma relação entre os sons das palavras e sua forma gráfica por meio da aprendizagem do nome e do som das letras. O reflexo dessa aprendizagem é evidenciado em suas formas de escrita inventadas. A fase seguinte, denominada alfabética completa, caracteriza-se pela habilidade para representar os sons das palavras por letras fonologicamente adequadas. Ao final, na fase alfabética consolidada, verifica-se a capacidade da criança para formar sílabas ou parte de sílabas das palavras por meio da junção de duas ou mais letras guiadas por sua fonologia (Cardoso-Martins \& Corrêa, 2008).

Outra forma de compreensão da aquisição da escrita e comumente associada à aprendizagem da leitura foi proposta por Frith (1986), que considera que a aprendizagem e o domínio da leitura e escrita são expressos por três fases. A fase logográfica ocorre no início dessa aprendizagem, quando a criança visualiza a palavra como se fosse um desenho e por meio de uma visão global a incorpora de forma gradativa, pois a escolha e a ordem das letras estão em dissonância aos sons da fala. Posteriormente, por meio do contato com as normas que determinam a aprendizagem do sistema escrito, ela passa para o estágio alfabético, no qual ocorre a codificação dos sons da fala com a escrita. A criança passa a discriminar palavras inteiras, ao invés de letras isoladas. No início, a escrita costuma apresentar alguns erros e de forma lenta e gradual, se torna satisfatória. O último estágio corresponde à fase ortográfica, no qual é desenvolvida a estratégia lexical, compreendendo que existem irregularidades nas relações que permeiam os fonemas e grafemas. Isso ocorre com o subsídio da memória, que favorece com que a criança passe a escrever de forma mais rápida e fluente, sem depender somente da estratégia fonológica (Capovilla, Varanda \& Capovilla, 2006; Sousa \& Maluf, 2004).

Independentemente do modelo adotado, grande parte dos estudos com consciência fonológica tem focalizado a sua relação com a leitura 
(Mota \& Santos, 2009; Pestun, 2005; Puliezi \& Maluf, 2012; Suehiro \& Santos, 2015). Em número menor, há estudos que exploram sua associação com a linguagem escrita, sendo alguns deles descritos na sequência.

O estudo de delineamento experimental desenvolvido por Carvalho (2010) objetivou investigar os efeitos de intervenções envolvendo a consciência fonológica em alunos com dificuldades de aprendizagem da escrita. A amostra contou com 18 estudantes do $3^{\circ}$ e $4^{\circ}$ ano do fundamental I, com idade média de oito anos e dois meses. Os alunos foram divididos em dois grupos. No Grupo Controle, aplicou-se o pré-teste e o pós-teste, tendo como instrumentos testes que avaliavam a consciência fonológica, a escrita e a leitura. Já o Grupo Experimental foi submetido além do pré e pós-testes, a intervenções com tarefas e jogos que visavam estimular a consciência fonológica. Ao final da pesquisa, a autora concluiu que o desenvolvimento e a aplicação de atividades que estimulem o exercício da relação entre fonemas e grafemas nos primeiros anos escolares é importante. As intervenções se mostraram como uma maneira eficaz para prevenir o baixo rendimento escolar em decorrência do pouco domínio da escrita e leitura.

Resultado semelhante foi encontrado por González, Cuetos, Vilar e Uceira (2015), em um estudo de delineamento experimental e longitudinal, desenvolvido durante três anos. A pesquisa contou com a participação de 271 alunos de 4 a 5 anos de idade, provindos de quatro escolas públicas espanholas. Para o grupo controle (GC) $(n=$ 133), foram aplicados testes de inteligência e ditados como provas de avaliação da escrita. Ao grupo experimental $(G E)(n=138)$, além dos testes e ditados, foi aplicado um programa de intervenção contendo tarefas elaboradas para estimular a consciência fonológica. No geral, os estudantes do GE demonstraram um desempenho superior em escrita quando comparados aos do GC. Esse resultado foi atribuído pelos pesquisadores em função da automatização das regras de conversão dos fonemas em grafemas, motivadas pelas intervenções envolvendo a consciência fonológica, que contribuíram para a escrita correta das palavras durante a transcrição dos ditados.

Além dessas, outras pesquisas também demonstraram que intervenções contendo tarefas envolvendo essas habilidades favorecem a aprendizagem e o domínio da escrita, tanto de cunho preventivo, como remediativo (Capovilla et al., 2004; Dambrowski, Martins, Theodoro \& Gomes, 2008). Investigações recentes, com o objetivo de correlacionar a consciência fonológica e a escrita, também se mostram de grande valia, por aferirem evidências de associação entre estas duas habilidades.

Em um estudo Iongitudinal, Andrade, Andrade e Capellini (2014) correlacionaram a leitura, escrita e a consciência fonológica e compararam as médias obtidas nestas habilidades no início do $2^{\circ} \mathrm{e}$ 
$3^{\circ}$ anos do ensino fundamental. A pesquisa contou com uma amostra de 45 alunos, sendo 29 meninos e 16 meninas, que no início do estudo apresentaram uma média de idade de sete anos e quatro meses. Os participantes foram classificados em grupos, sendo os alunos sem dificuldades (Grupo Controle - GC, $\mathrm{n}=32$ ) e com dificuldades (Grupo de Risco - GR, $\mathrm{n}=13$ ), conforme os resultados obtidos por uma avaliação diagnóstica inicial. Com foco na primeira aplicação, os resultados indicaram uma correlação alta para a consciência fonológica e a escrita $(r=0,63 ; p<0,001)$. Quanto às diferenças entre os grupos, o GC apresentou maiores médias em escrita $(M=34,12 ; p<0,001)$ e consciência fonológica $(M=38,22$; $\mathrm{p}<0,001)$ em comparação aos componentes do $\mathrm{GR}$ ( $\mathrm{M}$ escrita = 20,15; $M$ consciência fonológica $=31,38$; ambos $p<0,001)$. Diante dos resultados, os autores sugeriram o desenvolvimento de um modelo de intervenção precoce, baseado no perfil linguístico e cognitivo do aluno para contribuir com a aprendizagem da escrita.

Valendo-se de uma pesquisa correlacional, Justi e Roazzi (2012) investigaram a contribuição da consciência fonológica com a escrita em 94 alunos matriculados no $4^{\circ}$ ano do ensino fundamental (M idade $=$ nove anos e três meses). Para avaliar a escrita foi utilizado o Subteste de Escrita do Teste de Desempenho Escolar (TDE) e, para a aferição da consciência fonológica, a Tarefa de Subtração de Fonemas (SF) e a Tarefa de Spoonerismo (Spoon). As análises mostraram um índice de correlação de 0,32 entre a escrita e SF e de 0,50 entre escrita e o Spoon.

Por sua vez, Melo e Correa (2013) desenvolveram um estudo longitudinal e correlacional entre a consciência fonológica e escrita em 38 jovens e adultos ( $M$ idade $=38$ anos e dois meses; sendo 11 homens e 27 mulheres), matriculados em turmas de alfabetização para Jovens e Adultos (EJA). A escolha desse público-alvo se deu com o pressuposto que os alunos do EJA utilizam a consciência fonológica no início da aprendizagem da leitura e escrita de maneira similar a das crianças. A consciência fonológica foi avaliada por meio da rima, aliteração e segmentação e a escrita por meio de um ditado. Os resultados apontaram para a existência de correlações fracas a moderadas entre a consciência fonológica e a escrita, sendo rima $r=$ 0,34 ; segmentação $r=0,30$ e aliteração $r=0,40$.

Diante do exposto, percebe-se a importância de estudos que mostrem a relação entre a consciência fonológica e a escrita, de modo que aspectos ligados à consciência fonológica possam ser mais bem trabalhados em sala de aula, com o intuito de se prevenir possíveis dificuldades no aprendizado da escrita, mostrando a importância desta habilidade para a aquisição da escrita, assim como a possível prevenção de futuras dificuldades de aprendizagem. Sob essa perspectiva, este estudo objetivou investigar a associação entre a consciência fonológica e a escrita, assim como comparar os níveis 
de consciência fonológica, considerando as variáveis ano escolar, região de moradia e sexo dos participantes.

\section{Método}

Participantes

Participaram deste estudo 317 alunos de escolas públicas do $3^{\circ}(\mathrm{n}=$ $107), 4^{\circ}(n=110)$ e $5^{\circ}(n=100)$ anos do ensino fundamental, sendo 136 meninos e 181 meninas, com idades entre sete e doze anos $(M=9,29$ anos; $D P=1,147)$. A pesquisa é resultante de um projeto em parceria entre instituições universitárias das cinco regiões do Brasil. Assim, a coleta de dados ocorreu nas cidades onde essas instituições se localizavam, a saber, uma cidade do interior do Estado do Paraná, na região Sul $(n=56)$; a capital do Estado de Mato Grosso do Sul, na região Centro Oeste $(n=62)$; uma cidade do interior de São Paulo, na região Sudeste $(n=60)$; uma cidade do interior do Estado da Bahia, na região Nordeste $(n=71)$, na capital do Estado do Amazonas, na região Norte $(n=68)$.

\section{Instrumentos}

Escala de Avaliação da Escrita - EAVE (Sisto, 2005)

Este instrumento é composto por 55 palavras, que devem ser escritas pela criança após serem ditadas uma única vez e mede a capacidade de representação dos fonemas (sons) por meio do ditado. Das 55 palavras ditadas, 42 possuem certos níveis de dificuldade, sendo elas: encontro consonantal (It, $\mathrm{mp}, \mathrm{nd}, \mathrm{nt}, \mathrm{rc}, \mathrm{rs}, \mathrm{rt}, \mathrm{st}$ ), dígrafo (ch, Ih, nh, qu, rr, ss), sílaba composta (br, dr, gr, tr) e sílaba complexa (ao, ci, sa). Algumas palavras ditadas na EAVE devem ser escritas com a primeira letra maiúscula, sendo sinalizadas pelo aplicador antes de ditá-las. Na correção, é atribuído um ponto para cada palavra escrita de maneira incorreta e zero para a palavra redigida de forma correta, obtendo ao final um escore máximo de 55 pontos e mínimo de zero. São considerados erros as palavras escritas com sua forma gráfica incorreta, sem acentuação e as que não foram escritas com a primeira letra maiúscula, conforme as instruções do aplicador. São pontuadas também as palavras que são puladas pelo examinando. Estudos iniciais do autor do instrumento apontam para a adequação de suas propriedades psicométricas, tanto em termos de suas evidências de validade como sua estimativa de consistência interna. 
Roteiro de Avaliação da Consciência Fonológica - RACF (Santos, 1996).

Este instrumento é de aplicação individual e se divide em três blocos, sendo que cada um contém duas palavras como modelo, seguidas de outras três como alternativas de respostas, usadas para o treino dos respondentes. Em seguida aos modelos, há cinco itens em cada bloco, sendo que o primeiro deles tem o intuito de avaliar a percepção do fonema (som) inicial (aliteração), o segundo o som final (rima) e o terceiro bloco o som do meio das palavras. A correção é realizada com a atribuição de 1 ponto para cada acerto e zero para cada erro, sendo possível atingir um total mínimo de zero e o máximo de 15 pontos.

Quanto às propriedades psicométricas, o estudo da autora the conferiu evidência de validade experimental. Posteriormente, em outra pesquisa, foi verificada evidência de validade convergente com o teste de Cloze que avalia a compreensão de leitura $(r=-0,84 ; p<$ 0,001) (Suehiro \& Santos, 2012). A estimativa de precisão foi calculada para esta amostra e identificada como adequada $(a=0,72)$.

\section{Procedimentos}

Primeiramente, com a aprovação das instituições, o projeto foi submetido e aprovado por Comitê de Ética e combinadas as aplicações com os pesquisadores de cada região do Brasil. Os dados foram coletados por alunos de graduação em Psicologia, especialmente treinados para a tarefa e sob supervisão de um dos pesquisadores. Foi solicitado aos pais ou responsáveis pelos estudantes que assinassem o Termo de Consentimento Livre e Esclarecido (TCLE), os quais foram entregues previamente à aplicação dos instrumentos. A EAVE foi aplicada de forma coletiva nas salas de aula de cada escola e o RACF de forma individual, sendo que cada um deles levou aproximadamente 15 minutos para ser realizado. Vale ressaltar que o banco de dados contava com um número superior de participantes, porém nem todos responderam o RACF. Assim, o critério de inclusão para esta pesquisa se pautou em incluir somente os protocolos dos alunos que haviam respondido ambos os instrumentos.

\section{Análise de dados}

As análises dos dados foram realizadas no Software Statistical Package for the Social Sciences (SPSS), versão 22.0. Utilizou-se a Estatística Descritiva para se obter a média e desvio padrão dos alunos para os dois instrumentos. Não houve dados faltosos, nem omissos, visto que em ambas as medidas as respostas em branco ou 
ausentes foram consideradas como erros. Após determinação da normalidade dos dados relativos aos escores dos dois instrumentos aplicados, optou-se pelas provas de estatística paramétrica, calculando-se a correlação entre os instrumentos, bem como sua magnitude por meio do teste de correlação de Pearson. Para averiguar o desempenho nas variáveis ano escolar e região de moradia dos participantes, aplicou-se a Análise de Variância (ANOVA) e a prova post hoc de Tukey. Por fim, aplicou-se o Teste t de Student para verificar o desempenho para o fator sexo.

\section{Resultados}

A análise descritiva dos instrumentos foi realizada a fim de encontrar as médias dos alunos para os dois instrumentos. A média para a EAVE foi de $26,07(n=317 ; \mathrm{DP}=14,10)$. Já o RACF apresentou uma média de $11,78(n=315 ; \mathrm{DP}=2,66)$.

$O$ teste de correlação de Pearson apontou para uma relação estatisticamente significativa e negativa entre o RACF e a EAVE ( $r=-$ $0,30 ; p=0,001)$. Apesar de ambas as habilidades apresentarem uma relação de magnitude fraca (Dancey \& Reidy, 2006), o resultado sugere que o bom desempenho em consciência fonológica se reflete em menor número de erros de escrita. Nas Tabelas 1 e 2 são apresentados os resultados para a variável ano escolar na EAVE e no RACF. Em ambos os testes foram aplicadas a Análise de Variância ANOVA e a prova post hoc de Tukey.

Tabela 1

Post hoc de Tukey para dos escores da EAVE por ano escolar

\begin{tabular}{lccc}
\hline Ano Escolar & $\mathrm{n}$ & \multicolumn{3}{c}{ Médias da EAVE } \\
& & 1 & 2 \\
\hline Quinto Ano & 100 & 22,10 & \\
Quarto Ano & 110 & 23,33 & \\
Terceiro Ano & 107 & & 32,60 \\
$P$ & & 0,782 & 1 \\
\hline
\end{tabular}

As diferenças para a EAVE para variável ano escolar obtiveram valor estatisticamente significativo $[F(2,314)=19,559 ; \mathrm{p}=0,001]$. Aplicada a prova post hoc de Tukey, houve a formação de dois grupos, em que a média do quinto ano $(M=22,10)$ foi menor que a apresentada pelo quarto $(M=23,33)$ e terceiro ano $(M=32,60)$. Esses escores apontam um melhor desempenho em escrita para os alunos do quinto ano em relação aos do quarto e terceiro ano, visto que a EAVE pontua erros. 
Tabela 2

Post hoc de Tukey para comparação entre os escores do RACF por ano escolar

\begin{tabular}{lccc}
\hline Ano Escolar & $\mathrm{n}$ & \multicolumn{2}{c}{ Médias do RACF } \\
& \multicolumn{1}{c}{1} & 2 \\
\hline Terceiro Ano & 105 & 10,94 \\
Quarto Ano & 110 & & 11,95 \\
Quinto Ano & 100 & & 12,48 \\
$P$ & & 1 & 0,308 \\
\hline
\end{tabular}

A comparação entre os anos escolares para as pontuações do RACF na variável ano escolar também apresentou valor estatisticamente significativo $[F(3,312)=9,358 ; p=0,001]$. O teste post hoc de Tukey separou os escores em dois grupos em que o terceiro ano $(M=$ $10,94)$ aparece com uma média inferior em relação ao quarto ( $M=$ $11,95)$ e quinto ano $(M=12,48)$. A seguir são apresentadas as Tabelas 3 e 4 , referentes à variável região de moradia dos participantes.

Tabela 3

Post hoc de Tukey para comparação entre escores da EAVE por região de moradia dos participantes

\begin{tabular}{lccccc}
\hline Região & $\mathrm{n}$ & \multicolumn{5}{c}{ Médias da EAVE } \\
& & 1 & 2 & 3 & 4 \\
\hline Sudeste & 60 & 17,05 & & & \\
Sul & 56 & 20,57 & 20,57 & & \\
Centro Oeste & 62 & & 26,13 & 26,13 & \\
Nordeste & 71 & & & 31,69 & 31,69 \\
Norte & 68 & & & & 32,63 \\
$P$ & & 0,535 & 0,109 & 0,108 & 0,994 \\
\hline
\end{tabular}

A comparação dos resultados da EAVE para a variável região de moradia obteve valor estatisticamente significativo $[\mathrm{F}(4,312)=$ $17,925 ; p=0,001]$. O post hoc de Tukey separou os escores em quatro grupos, sendo que as crianças da região Sudeste $(M=17,05)$ evidenciaram um desempenho superior quando comparadas às crianças das outras regiões e a média dos alunos da região Norte ( $M$ $=32,63)$ foi inferior. 
Tabela 4

Post hoc de Tukey para comparação entre escores do RACF por região de moradia dos participantes

\begin{tabular}{lcccc}
\hline Região & $\mathrm{n}$ & \multicolumn{3}{c}{ Médias do RACF } \\
& & 1 & 2 & 3 \\
\hline Norte & 67 & 10,12 & & \\
Sudeste & 60 & & 11,50 & \\
Sul & 56 & & 11,54 & \\
Centro Oeste & 61 & & 12,13 & \\
Nordeste & 71 & & & 13,49 \\
$P$ & & 1 & 0,590 & 1 \\
\hline
\end{tabular}

As diferenças de médias do RACF para a variável regiões apresentaram valor estatisticamente significativo $[F(4,310)=$ $17,433 ; p=0,001]$. A análise dos escores permitiu a formação de três grupos em que as crianças da região Nordeste $(M=13,49)$ obtiveram a maior média e a região Norte $(M=10,12)$ a menor, quando comparadas com as médias das outras regiões de moradia.

Por fim, para avaliar a diferença de desempenho para a variável sexo aplicou-se o teste $\mathrm{t}$ de Student. Os resultados apontaram para um melhor desempenho para as meninas em comparação aos meninos para a EAVE (Meninas: $M=25,33$; Meninos: $M=27,06 ; t=1,083 ; p$ $=0,280$ ) e para o RACF (Meninas: $M=11,81$; Meninos: $M=11,76 ; t$ $=-0,165 ; p=0,869)$. No entanto, as análises não indicaram diferenças significativas para o desempenho dos alunos nessa variável, em ambos os sexos nos dois instrumentos com $p>0,05$, como o indicado.

\section{Discussão dos resultados}

A análise dos resultados indicou a correlação entre a consciência fonológica e a escrita. Apesar de fraca, tal relação alude à contribuição dessa habilidade metalinguística para a escrita, principalmente durante a sua aquisição. Nesse sentido, Costa (2013) identificou em pesquisa com crianças pré-escolares e do $1^{\circ}$ ano do fundamental, que os alunos com altos níveis em consciência fonológica demonstraram melhor desempenho em escrita. Valente e Martins (2012) destacaram que discernir o som das letras favorece o estabelecimento das relações entre fonemas e grafemas, o que contribui para a aprendizagem da escrita, em especial em línguas classificadas como transparentes, como é o caso do português, em que há grande correspondência entre letra e som (Hagen, Miranda, \& Mota, 2010). Pestun, Omote, Barreto, e Matsuo (2010), ainda averiguaram que intervenções em consciência fonológica em alunos 
do $1^{\circ}$ ano apresentaram resultados positivos no desempenho em escrita. Resultados similares foram encontrados por Costa (2013) e Rego e Bryant (1993).

Para a variável ano escolar, os resultados evidenciaram um melhor desempenho tanto em escrita quanto em consciência fonológica para os alunos do $5^{\circ}$ ano em comparação aos do $3^{\circ}$ ano. Este resultado pode ser compreendido em congruência com as reflexões de autores que sugerem que, no decorrer da passagem dos anos escolares, 0 aluno passa por diversas mudanças relativas à aquisição de novas experiências e do amadurecimento de suas estruturas cognitivas (Pestun, 2005; Suehiro \& Magalhães, 2014).

Especificamente no que se refere à escrita, Sisto e Fernandes (2004) asseveram que há grande probabilidade de que as regras da linguagem escrita não estejam incorporadas ao repertório do aluno dos primeiros anos escolares, levando-os a cometerem alguns erros. Resultados semelhantes também foram encontrados por Suehiro e Hohlenwerger (2014) que também detectaram que com a passagem dos anos escolares, o aluno passa a cometer menos erros, apresentando um domínio maior na escrita.

Em relação ao desempenho superior em consciência fonológica, pontuado pelos alunos do $5^{\circ}$ ano, Pestun (2005) considera o avanço do ensino formal como um fator associado ao maior nível de desempenho. No mesmo sentindo, Costa (2013) ressalta a contribuição da aplicação de tarefas que se propunham a estimular a consciência fonológica em programas de intervenção, os quais favorecem, por exemplo, com que alunos pré-silábicos passem para o nível silábico. Considera-se, ainda, que tarefas com foco nos componentes sonoros e seus segmentos referentes à consciência fonológica refletem na passagem dos níveis de escrita (Costa, 2013; Pestun, 2005).

Os resultados para a variável região de moradia sinalizaram melhor desempenho para a região Sudeste na EAVE e níveis inferiores para a região Norte em ambos os instrumentos. Tais resultados são coerentes com a avaliação de desempenho geral dos alunos do ensino fundamental com base na Prova Brasil e no Sistema de Avaliação da Educação Básica (SAEB) (Costa, 2010; Klein, Fontanive, Restani \& Telles, 2013). As médias apontaram para um melhor desempenho para os alunos provindos da região Sudeste em comparação aos alunos da região Norte e Nordeste, o que corrobora apenas em parte com a presente pesquisa. Importante salientar que nos escores da medida de consciência fonológica (RACF) os alunos da região Nordeste apresentaram um melhor desempenho em relação às demais regiões. Esses dados indicam a necessidade de novas investigações, em especial nas regiões Norte e Nordeste, a fim de identificar e ter mais elementos sobre a questão. A razão dessas diferenças poderia ser atribuída a uma miríade de aspectos 
eventualmente relacionados à formação dos professores, ao acompanhamento e apoio técnico às escolas, entre outras. Ainda, nesse sentido, seria necessário saber mais sobre as propostas curriculares e se há intervenções de cunho preventivo e remediativo que se adequem às necessidades pedagógicas dos alunos matriculados nos primeiros anos escolares (Ministério da Educação, 2014).

Apesar de que os resultados não indicaram diferenças significativas para a variável sexo, neste estudo, pesquisas realizadas por Suehiro e Hohlenwerger (2014), com o objetivo de avaliar a escrita por meio da EAVE, identificaram desempenho superior para as meninas, expressos em um número menor de erros para o instrumento em comparação aos meninos, o que corrobora com a literatura. No que tange à consciência fonológica, Andreazza-Balestrin, Cielo, Volcão e Lasch (2012) conferiram que as meninas obtiveram desempenho superior aos meninos em tarefas envolvendo a consciência fonológica, tais como, segmentação silábica, aliteração, detecção da sílaba do meio e rima. Outros estudos realizados com o objetivo de investigar 0 desempenho em consciência fonológica não detectaram diferenças significativas entre os sexos (Meneses, Lozi, Souza \& AssencioFerreira, 2004; Moura, Cielo \& Mezzomo, 2009). Em razão de resultados incongruentes detectados na literatura, sugere-se a necessidade de novas investigações acerca do desempenho em escrita e consciência fonológica em meninos e meninas.

\section{Considerações finais}

Considera-se relevante a iniciativa de promover estudos com o objetivo de averiguar o nível de dificuldade nas habilidades linguísticas e metalinguísticas, representadas neste estudo pela escrita e a consciência fonológica, em alunos matriculados nos primeiros anos de alfabetização, antes até, como na pré-escola, principalmente com relação à consciência fonológica. São concebidos inúmeros fatores relacionados à aprendizagem e domínio da escrita, o que justifica investigar os elementos que auxiliam na sua aquisição. Assim, aferir a relação entre o aspecto cognitivo, aqui representado pela consciência fonológica, pode auxiliar na identificação de diagnósticos sobre possíveis dificuldades em escrita, bem como destacar sua contribuição positiva, assim como prevenir problemas relacionados, não só à aprendizagem da escrita, mas à aprendizagem de modo geral.

Enfatiza-se aqui a promoção de ações preventivas, por favorecerem o desenvolvimento do aluno antes mesmo que problemas expressos por dificuldades em escrita ocorram. Assim, destaca-se a contribuição potencial da avaliação psicológica aliada à educação por fomentar a 
construção de instrumentos com o intuito de avaliar a escrita e os elementos relacionados a esta habilidade. Portanto, essas investigações visam subsidiar esses dois campos do conhecimento, no que se refere ao aprimoramento das propriedades psicométricas dos instrumentos, assim como possibilitar a contextualização e elaboração de intervenções que favoreçam o processo de ensino e aprendizagem.

Ressalta-se, ainda, a importância para a investigação dos fatores relativos ao ano escolar e à região de moradia. A avaliação por ano escolar buscou conferir se os alunos dos anos posteriores apresentavam maior domínio da escrita e apropriação dos níveis que compõem a consciência fonológica em comparação aos alunos dos primeiros anos escolares, o que pôde ser aferido nesse estudo. Por fim, abarcar as cinco regiões brasileiras possibilita a mensuração e visualização das diferenças no desempenho desses alunos, servindo como base para a reflexão sobre a forma com que a educação básica tem sido disponibilizada na rede de ensino público. Outro fator ligado a essa variável se refere à grande concentração de estudos envolvendo essa temática nas regiões Sul e Sudeste, também conferido por Oliveira, Cantalice, Joly e Santos (2006), o que restringe, em partes, o conhecimento sobre aspectos relativos à educação em outras regiões do Brasil.

Atrelada aos referidos aspectos aqui citados, associa-se também a produção de pesquisas nessa área como uma forma de avaliar e promover discussões acerca da melhoria da qualidade de ensino, a reflexão sobre os sistemas de avaliação, a formação dos profissionais que atuam no contexto escolar, os recursos investidos nas áreas de tecnologias e produção científica no campo da educação, previstos pelo Plano Nacional de Educação - PNE (Ministério da Educação, 2014). Reconhece-se que este estudo não alcança todos esses aspectos, mas faz um recorte do desempenho em escrita e em consciência fonológica de alunos de todas as regiões do país. Fornece, também, um incentivo para a formulação de futuras pesquisas abarcando, tanto os construtos pesquisados, como de outras variáveis associadas a esta temática.

Por fim, sabe-se que todos os estudos possuem limitações, neste uma delas foi o número reduzido de participantes, considerando o universo de alunos do ensino fundamental no país. Além disso, não houve controle do nível das escolas envolvidas em termos do desempenho de seus alunos nas provas nacionais, regularmente aplicadas pelo Ministério da Educação. Ainda, sugere-se que outros construtos sejam atrelados aos aqui pesquisados, como, por exemplo, atenção, memória e reconhecimento de palavras, com a intenção de, considerando-se a fraca magnitude da correlação entre a consciência fonológica e a escrita, com o intuito de identificar quais habilidades 
estão mais estreitamente envolvidas no processo de aquisição da linguagem escrita.

\section{Referências}

Andrade, O. V. C. A., Andrade, P. E., \& Capellini, S. A. (2014). Caracterização do perfil cognitivo-linguístico de escolares com dificuldades de leitura e escrita. Psicologia: Reflexão e Crítica, 27(2), 358-367. doi: 10.1590/1678-7153.201427217

Andreazza-Balestrin, C., Cielo, C. A., Volcão, C. L. \& Lasch, S. S. (2012). Abilities in phonological awareness: differences in boys and girls' performance. Revista CEFAC, 14(4), 669-676. doi: $10.1590 / S 1516-18462011005000070$

Araujo, M. R., \& Minervino, C. A. S. M. (2008). Avaliação cognitiva: leitura, escrita e habilidades relacionadas. Psicologia em Estudo, 13(4), 859-865. doi: 10.1590/S1413-73722008000400024

Capovilla, A. G. S., Gutschow, C. R. D., \& Capovilla, F. C. (2004). Habilidades cognitivas que predizem competência de leitura e escrita. Psicologia: Teoria e Prática, 6(2), 13-26. Recuperado de http://pepsic.bvsalud.org/pdf/ptp/v6n2/v6n2a02.pdf

Capovilla, F. C., Varanda, C., \& Capovilla, A. G. S. (2006). Teste de Competência de Leitura de Palavras e Pseudopalavras: normatização e validação. Psic: revista da Vetor Editora, 7(2), 47-59. Recuperado de http://pepsic.bvsalud.org/scielo.php?pid=S1676$73142006000200007 \&$ script $=$ sci_arttext\&tIng $=e s$

Cardoso-Martins, C. (2013). A consciência fonológica e a aprendizagem inicial da leitura e da escrita. Cadernos de pesquisa, (76), 41-49. Recuperado de http://publicacoes.fcc. org.br/ojs/index.php/cp/article/view/105 3

Cardoso-Martins, C., \& Correa, M. F. (2008). O desenvolvimento da escrita nos anos pré-escolares: Questões acerca do estágio silábico. Psicologia. Teoria e pesquisa, 24(3), 279-286. Recuperado de http://cat.inist.fr/ ?aModele $=$ afficheN\&cpsidt $=20874450$

Cárnio, M., S., Pereira, M., B., Alves, D., C., \& Andrade, R., V. (2011). Letramento escolar de estudantes de $1^{a}$ e $2^{a}$ séries do ensino fundamental de escola pública. Revista da Sociedade Brasileira de Fonoaudiologia, 16(1), 1-8. doi: 10.1590/S151680342011000100003

Carvalho, L. M. D. M. (2010). Consciência fonológica e sucesso na aprendizagem da leitura e da escrita: melhor prevenir do que remediar. (Tese de Doutorado). Universidade Católica de São Paulo, São Paulo, Brasil. 
Costa, A. C. (2013). Consciência fonológica: relação entre desenvolvimento e escrita. Letras de hoje, 38(2), 137-153. Recuperado de http://revistaseletronicas.pucrs.br/ojs/index.php/fale/article/vie wArticle/14095

Costa, C. C. R. C. D. (2010). O Ideb como indicador da qualidade da educação brasileira: o caso da região Sudeste. e-Xacta, 3(2), 323. Recuperado de http://revistas.unibh.br/index.php/dcet/article/view/297

Dambrowski, A. B., Martins, C. L., Theodoro, J. D. L. \& Gomes, E. (2008). Influência da consciência fonológica na escrita de préescolares. Revista CEFAC-Speech, Language, Hearing Sciencesand Education Journal, 10(2), 175-81. doi: 10.1590/S1516-18462008000200006

Dancey, C. P., \& Reidy, J. (2006). Estatística sem matemática para a psicologia: usando SPSS para Windows. Porto Alegre: Artmed.

Fletcher, J. M., Lyons, G. R., Fuchs, L. S., \& Barnes, M. A. (2009). Transtornos de aprendizagem: da identificação à intervenção. Porto Alegre: Artmed.

Frith, U. (1986). A developmental framework for developmental dyslexia. Annals of dyslexia, 36(1), 67-81. doi: 10.1007/BF02648022

González, R. M., Cuetos, F., Vilar, J. \& Uceira, E. (2015). Efectos de la intervención em conciencia fonológica y velocidad de denominación sobre elaprendizaje de la escritura. Aula Abierta, 43(1), 1-8. doi: 10.1016/j.aula.2014.06.001

Hagen, V., Miranda, L. C. \& Mota, M. M. P. E. D. (2010). Consciência morfológica: um panorama da produção científica em línguas alfabéticas. Psicologia: Teoria e Prática, 12(3), 135-148. Recuperado de http://pepsic.bvsalud.org/pdf/ptp/v12n3/v12n3a11.pdf

Justi, C. N. G. \& Roazzi, A. (2012). A contribuição de variáveis cognitivas para a leitura e a escrita no português brasileiro. Psicologia: Reflexão e Crítica, 25(3), 605-614. Recuperado de http://www.scielo.br/pdf/prc/v25n3/v25n3a21.pdf

Klein, R., Fontanive, N., Restani, A. L. \& Telles, M. C. (2013). O desempenho dos alunos da Fundação Bradesco: uma comparação com os resultados do Saeb. Estudos em Avaliação Educacional, 19(41), 499-515. doi: $10.18222 /$ eae 194120082074

Lopes, E. J., Lopes, R. F. F. \& Galera, C. A. (2005). A memória de trabalho viso-espacial em crianças de 7 a 12 anos. Estudos de Psicologia, 10 (2). 207-214). Recuperado de http://www.scielo.br/pdf/epsic/v10n2/a07v10n2

Melo, R. B. \& Correa, J. (2013). Consciência Fonológica e a aprendizagem da leitura e escrita por Adultos. Estudos e 
Pesquisas em Psicologia, 13(2), 460-479. Recuperado de http://www.e-

publicacoes.uerj.br/ojs/index.php/revispsi/article/view/8419

Meneses, M. S., Lozi, G. P., Souza, L. R., \& Assencio-Ferreira, V. J. (2004). Consciência fonológica: diferenças entre meninos e meninas. Rima (R), 8, 9. Recuperado de http://cefac.br/revista/revista63/Artigo\%202.pdf

Mezzomo, C. L., Mota, H. B., \& Dias, R. F. (2010). Desvio fonológico: aspectos sobre produção, percepção e escrita. Revista da Sociedade Brasileira de Fonoaudiologia, 15(4), 554-560. doi: 10.1590/S1516-80342010000400013

Ministério da Educação (2014). Plano Nacional de Educação - PNE 2014-2024. Recuperado de http://www.observatoriodopne.org.br/uploads/reference/file/43 9/documento-referencia.pdf

Moura, S. R. S., Cielo, C. A. \& Mezzomo, C. L. (2009). Consciência fonêmica em meninos e meninas. Revista da Sociedade Brasileira de Fonoaudiologia, 14(2), 205-11. Recuperado de http://www.scielo.br/pdf/rsbf/v14n2/11.pdf

Mota, M. M. P. E. \& Santos, A. A. A. (2009). O papel da consciência fonológica na leitura contextual medida pelo teste de Cloze. Estudos de psicologia, 14(3), 207-212. Recuperado de http://www.scielo.br/pdf/epsic/v14n3/a04v14n3

Oliveira, K. L., Cantalice, L. M. D., Joly, M. C. R. A. \& Santos, A. A. A. D. (2006). Produção científica de 10 anos da revista Psicologia Escolar e Educacional (1996/2005). Psicologia Escolar e Educacional, 10(2), 283-292. doi: 10.1590/S141385572006000200011

Pestun, M. S. V. (2005). Consciência fonológica no início da escolarização e o desempenho ulterior em leitura e escrita: estudo correlacional. Estudos de Psicologia, 10(3), 407-412. doi: 10.1590/S1413-294X2005000300009

Pestun, M. S. V., Omote, L. C., Barreto, D. C. \& Matsuo, T. (2010). Estimulação da consciência fonológica na educação infantil: prevenção de dificuldades na escrita. Psicologia Escolar e Educucacional, 14(1), 95-104. doi: 10.1590/S141385572010000100011

Pollo, T. C., Treiman, R. \& Kessler, B. (2015). Uma revisão crítica de três perspectivas sobre o desenvolvimento da escrita. Estudos de Psicologia, 32(3), 449-459. doi: 10.1590/0103$166 \times 2015000300010$

Puliezi, S., \& Maluf, M. R. (2012). A contribuição da consciência fonológica, memória de trabalho e velocidade de nomeação na aquisição inicial da leitura. Boletim Academia Paulista de Psicologia, 32(82), 213-227. Recuperado de 
http://repositoriodigital.academica.mx/jspui/handle/987654321 /278898

Rego, L., \& Bryant, P. (1993). The connections between phonological, syntactic and semantic skills and children's reading and spelling. European Journal of Psychology, 3, 235-246. doi: 10.1007/BF03174079

Santos, A. A. A. (1996). A influência da consciência fonológica na aquisição da leitura e da escrita. In F. F. Sisto, G. C. Oliveira, L. D. T. Fini, M. T. C. C. Souza, \& R. P. Brenelli (Orgs.), Atuação psicopedagógica e aprendizagem escolar (pp. 213-247). Petrópolis: Vozes.

Sisto, F. F., \& Fernandes, D. C. (2004). Dificuldades linguísticas na aquisição da escrita e agressividade. Psicologia Escolar e Educacional, 8(1), 75-84. doi: 10.1590/S141385572004000100009

Sisto, F. F. (2005). Escala de Avaliação da Escrita (EAVE). Relatório técnico. Universidade São Francisco, Itatiba, São Paulo.

Sousa, É. D. O. \& Maluf, M. R. (2004). Habilidades de leitura e de escrita no início da escolarização. Psicologia da Educação, (19), 55-72. Recuperado http://pepsic.bvsalud.org/pdf/psie/n19/n19a04.pdf

Souza, A. R. M. \& Sisto, F. F. (2001). Dificuldade de aprendizagem em escrita, memória e contradições. Psicologia Escolar e Educacional, 5(2), 39-47. doi: 10.1590/S141385572001000200005.

Suehiro, A. C. B. \& Hohlenwerger, V. L. (2014). Escrita em grupo de escolares. Avaliação Psicológica, 13(3), 391-398. Recuperado de http://pepsic.bvsalud.org/pdf/avp/v13n3/v13n3a11.pdf

Suehiro, A. C. B. \& Magalhães, M. M. D. S. (2014). Relação entre medidas de Avaliação da Linguagem Escrita em estudantes do Ensino Fundamental. Psico USF, 19(3), 489-498. doi.org/10.1590/1413-82712014019003011

Suehiro, A. C. B. \& Santos, A. A. A. (2015). Compreensão de leitura e consciência fonológica: evidências de validade de suas medidas. Estudos de Psicologia (Campinas), 32(2), 201-211. doi: 10.1590/0103-166X2015000200005

Valente, F. \& Martins, M. A. (2012). Competências metalinguísticas e aprendizagem da leitura em duas turmas do $1^{\circ}$ ano de escolaridade com métodos de ensino diferentes. Análise Psicológica, 22(1), 193-212. Recuperado de http://repositorio.ispa.pt/bitstream/10400.12/211/1/AP\%2022 $\% 281 \% 29 \% 20193-212$.pdf 
Acácia Aparecida Angeli dos Santos, Adriana Satico Ferraz, Thatiana Helena de Lima, Neide de Brito Cunha, Adriana Cristina Boulhoça Suehiro, Katya Luciane de Oliveira, Alexandra Ayach Anache, Iolete Ribeiro da Silva

\section{Endereço para correspondência:}

Acácia Aparecida Angeli dos Santos

Universidade São Francisco - USF

Rua Waldemar Cesar da Silveira, 105, CEP 13045-510, Campinas - SP, Brasil

Endereço eletrônico: acacia.angeli@gmail.com

\section{Adriana Satico Ferraz}

Universidade São Francisco - USF

Rua Waldemar Cesar da Silveira, 105, CEP 13045-510, Campinas - SP, Brasil

Endereço eletrônico: adrianasatico.as@gmail.com

\section{Thatiana Helena de Lima}

Universidade Federal da Bahia - UFBA

Rua Aristides Novis, 197, CEP 40.210-730, Salvador - BA, Brasil

Endereço eletrônico: thatianahlima@gmail.com

\section{Neide de Brito Cunha}

Universidade do Vale do Sapucaí - UNIVAS

Av. Pref. Tuany Toledo, 470, CEP 37550-000, Pouso Alegre - MG, Brasil

Endereço eletrônico: neidedebritocunha@gmail.com

\section{Adriana Cristina Boulhoça Suehiro}

Universidade Federal do Recôncavo da Bahia - UFRB

Av. Carlos Amaral, 1015, Cajueiro, CEP 44574-490, Santo Antônio de Jesus - BA, Brasil

Endereço eletrônico: dricbs@yahoo.com.br

\section{Katya Luciane de Oliveira}

Universidade Estadual de Londrina - UEL

Rodovia Celso Garcia Cid, Km 380, s/n, Campus Universitário, CEP 86057-970, Londrina - PR, Brasil

Endereço eletrônico: katyauel@gmail.com

\section{Alexandra Ayach Anache}

Universidade Federal de Mato Grosso do Sul - UFMS

Av. Costa e Silva, s/n, Bairro Universitário, CEP 79070-900, Campo Grande - MS, Brasil

Endereço eletrônico: alexandra.anache@gmail.com

\section{I olete Ribeiro da Silva}

Universidade Federal do Amazonas - UFAM

Av. General Rodrigo Octávio Jordão Ramos, 6.200, Setor Sul, Bloco X, Campus Universitário Coroado I, CEP 69080-900, Manaus - AM, Brasil

Endereço eletrônico: iolete.silva@gmail.com

Recebido em: 03/09/2016

Reformulado em: $21 / 11 / 2016$

Aceito em: $21 / 11 / 2016$

\section{Notas}

Financiamento: CNPq.

* Doutora em Psicologia Escolar e do Desenvolvimento Humano pela USP/SP. Professora Titular da Universidade São Francisco. Bolsista Produtividade do CNPq.

** Psicóloga. Mestranda em Psicologia do Programa de Pós-Graduação da Universidade São Francisco.

*** Doutora em Psicologia pelo Programa de Pós-Graduação da Universidade São Francisco. Professora Adjunta da Universidade Federal da Bahia.

**** Doutora em Psicologia. Mestre em Educação. Licenciada em Letras e Pedagogia. Docente do Mestrado em Educação da Universidade do Vale do Sapucaí e do Centro Paula Souza. 
***** Psicóloga. Doutora em Psicologia pelo Programa de Pós-Graduação Stricto Sensu em Psicologia da Universidade São Francisco, pós-doutoramento em Educação pela Faculdade de Educação da UNICAMP e docente da Universidade Federal do Recôncavo da Bahia - Santo Antônio de Jesus.

****** Psicóloga. Doutora em Educação FE/UNICAMP. Docente do Curso de Psicologia e do Programa de Pós-graduação em Educação da UEL. Bolsista Produtividade do CNPq.

$* * * * * * *$ Psicóloga. Doutora em Psicologia Escolar e do Desenvolvimento Humano pela USP/SP. Professora dos Programas de Pós-Graduação em Psicologia e em Educação da UFMS.

********* Mestre e Doutora em Psicologia pela Universidade de Brasília, Professora Associada da Universidade Federal do Amazonas (UFAM) e do Programa de Pós-Graduação em Psicologia/UFAM e Programa de Pós-Graduação em Educação/UFAM. 